\title{
Analysis of the Stereophonic LMS/Newton Algorithm and Impact of Signal Nonlinearity on Its Convergence Behavior
}

\author{
Harsha I. K. Rao, Student Member, IEEE, and Behrouz Farhang-Boroujeny, Senior Member, IEEE
}

\begin{abstract}
The strong cross-correlation that exists between the two input audio channels makes the problem of stereophonic acoustic echo cancellation (AEC) complex and challenging to solve. Recently, two new implementations of the LMS/Newton algorithm that uses a linear decorrelation technique were proposed. This method helps to mitigate the effect of the ill-conditioned problem on the convergence rate of the LMS/Newton adaptive algorithm. The complexity of these algorithms is significantly lower than the recursive least-squares (RLS) algorithm, which is known to provide excellent echo cancellation. Furthermore, unlike the various versions of the RLS algorithm, the LMS/Newton algorithm is more robust to numerical errors. It has also been suggested that applying nonlinearities to signals at the two audio channels will help to alleviate the misalignment problem of stereophonic AEC systems. Simulation studies reveal that application of certain classes of nonlinearities to the two-channel LMS/Newton algorithms helps to further reduce the misalignment but it also leads to an unexpected and significant reduction in the rate of convergence of the mean-square error. The contributions of this paper are twofold. First, we provide an analysis of the two-channel LMS/Newton algorithm that was proposed in our earlier work. Second, we provide a theoretical understanding for the appearance of the slow modes of convergence in the presence of nonlinearities and show that they can be resolved through a preprocessing step.
\end{abstract}

Index Terms-Acoustic signal processing, adaptive filters, nonlinearities, stereophonic systems, teleconferencing.

\section{INTRODUCTION}

$\mathbf{T}$ HE use of multichannel audio in teleconferencing systems is highly desirable as it enhances sound realism by providing the listener with spatial information to help distinguish possibly simultaneous talkers [1]. These teleconferencing systems must use acoustic echo cancelers to remove the undesired echoes that result from the coupling between the microphones and the loudspeakers [2], [3]. While multichannel acoustic echo cancellation (AEC) can be seen as a simple generalization of the well-known single-channel AEC, it is a far more complex and challenging problem to solve [4].

Manuscript received February 08, 2010; accepted August 26, 2010. Date of publication September 09, 2010; date of current version November 17, 2010. The associate editor coordinating the review of this manuscript and approving it for publication was Dr. Konstantinos Slavakis.

H. I. K. Rao was with the Department of Electrical and Computer Engineering, University of Utah, Salt Lake City, UT 84112 USA. He is now with HARMAN, Sandy, UT 84070 USA (e-mail: hrao@eng.utah.edu).

B. Farhang-Boroujeny is with the Department of Electrical and Computer Engineering, University of Utah, Salt Lake City, UT 84112 USA (e-mail: farhang@ece.utah.edu).

Digital Object Identifier 10.1109/TSP.2010.2074198
The fundamental problem of stereophonic AEC was addressed in [1], [4]. The setup of a stereophonic acoustic echo canceler as it exists in a typical teleconferencing system is shown in Fig. 1 [1], [4]. A transmission room is shown on the left, wherein two microphones are used to pick up the signals from a source $s(n)$ via two acoustic channels characterized by the room impulse responses $g_{1}(n)$ and $g_{2}(n)$. The stereophonic signals are transmitted to the loudspeakers in the receiving room. These loudspeakers are coupled to one of the microphones via the acoustic channels denoted by $h_{1}(n)$ and $h_{2}(n)$. A conventional acoustic echo canceler will try to model the acoustic paths in the receiving room using two finite-impulse response (FIR) adaptive filters, $\hat{h}_{1}(n)$ and $\hat{h}_{2}(n)$. The two adaptive filters will use the input signals $x_{1}(n)$ and $x_{2}(n)$ to produce an estimate of the echo picked up by the microphone. If $d(n)$ denotes the echo picked up by the microphone and $y(n)$ is its estimate, then the difference between $d(n)$ and $y(n)$ should produce a residual echo signal $e(n)$ close to zero. We may note that the inputs $x_{1}(n)$ and $x_{2}(n)$ are derived from the same source $s(n)$ and hence are highly correlated. The strong cross-correlation between $x_{1}(n)$ and $x_{2}(n)$ can create problems in implementing adaptive algorithms.

The residual error signal $e(n)$ is given by

$e(n)=\left[\mathbf{h}_{1}(n)-\hat{\mathbf{h}}_{1}(n)\right]^{T} \mathbf{x}_{1}(n)+\left[\mathbf{h}_{2}(n)-\hat{\mathbf{h}}_{2}(n)\right]^{T} \mathbf{x}_{2}(n)$

where $\mathbf{h}_{i}(n)$ and $\hat{\mathbf{h}}_{i}(n), i=1,2$, are $L$-dimensional vectors of the near-end room echo paths and the adaptive filter coefficients, respectively, and $\mathbf{x}_{i}(n)=\left[x_{i}(n), x_{i}(n-1), \ldots, x_{i}(n-L+\right.$ 1) $]^{T}, i=1,2$ are vectors comprising the $L$ most recent loudspeaker signal samples, with superscript $T$ denoting the transpose operator. Furthermore, the mismatch between the modeling adaptive filters $\hat{\mathbf{h}}(n)=\left[\hat{\mathbf{h}}_{1}^{T}(n) \hat{\mathbf{h}}_{2}^{T}(n)\right]^{T}$ and the true near-end room echo paths $\mathbf{h}(n)=\left[\mathbf{h}_{1}^{T}(n) \mathbf{h}_{2}^{T}(n)\right]^{T}$ is known as the misalignment. The normalized misalignment is computed as

$$
\eta(n)=\frac{\|\mathbf{h}(n)-\hat{\mathbf{h}}(n)\|^{2}}{\|\mathbf{h}(n)\|^{2}} .
$$

An important result derived in [4] was that the misalignment problem is much greater in the two-channel setup due to the strong cross-correlation effects. While it is important that an adaptive filtering algorithm drives the residual error signal $e(n)$ to zero as quickly as possible, the reduction of the misalignment is also vital in the context of stereophonic AEC. It has been well studied in [1] and [4] that if the misalignment is large, any change in the far-end room echo paths $g_{1}(n)$ and $g_{2}(n)$ can 
drastically alter $e(n)$. This occurs frequently as different people may speak in the far-end room.

An exclusive maximum (XM) selective adaptation of filter taps was proposed in [5] to improve the convergence rate of several popular adaptive algorithms. The leaky extended leastmean-square (XLMS) algorithm of [6] also aims to achieve an improvement in the convergence rate. However, its complexity is twice that of a normalized least-mean-square (NLMS) algorithm.

Recently, we proposed a stereophonic extension [7] of the fast LMS/Newton algorithm of [8]. This algorithm makes use of the orthogonalization property of the two-channel lattice predictor [7]. The use of this simple linear decorrelation technique helps to significantly improve the convergence behavior of the LMS/Newton algorithm. The input signals $x_{1}(n)$ and $x_{2}(n)$ are modeled as autoregressive (AR) processes of order $M \ll L$. Thus, the computational complexity remains close to that of the NLMS algorithm.

The use of nonlinearities has been shown to successfully reduce the interchannel coherence between the signals in the two channels of the acoustic echo canceler, thereby reducing the misalignment problem [4]. While the use of a nonlinear (NL) preprocessor in conjunction with the two-channel LMS/Newton algorithms of [7] will further reduce the misalignment, it has been observed that there is a significant decrease in the convergence rate of the mean-square error (MSE). It is well known that the LMS/Newton algorithm is governed by a single mode of convergence [9]. Hence, the appearance of the slow modes in the learning curves of the LMS/Newton algorithm is highly uncharacteristic for this particular class of adaptive filtering algorithms.

Extensive computer simulations reveal that this observed phenomena is inherent in other adaptive algorithms as well, including the XM-NLMS algorithm of [5] and the leaky XLMS algorithm of [6]. In recent years, papers on stereophonic AEC have primarily focussed on presenting the improvement in misalignment and even from these results [5], one can notice the slow modes of convergence. It must be noted that algorithms such as the NLMS and its variants inherently suffer from slow modes of convergence in the mean-square error. Hence, the appearance of additional slow modes in the presence of nonlinearities may not appear obvious and, thus, has remained unnoticed. Moreover, nonlinearities play a greater role in improving the misalignment obtained by these algorithms and hence the difference in the convergence in the MSE is not that significant.

In this paper, we will highlight the impact of nonlinearities on the convergence behavior of the two-channel LMS/Newton algorithm of [7]. First, we present an analysis of the two-channel LMS/Newton algorithm. This will lay the framework to the- oretically analyze the effect of nonlinearities on its MSE performance.

The rest of this paper is organized as follows. In Section II, we present a summary of the two-channel LMS/Newton adaptive algorithm proposed in [7]. Analysis of this algorithm is provided in Section III. In Section IV, we will examine the impact of signal nonlinearity on the convergence behavior of the two-channel LMS/Newton algorithm. We will present a number of simulation results that will verify our theoretical findings. We will also propose a preprocessing technique that will resolve the problem of slow convergence. Conclusions are drawn in Section V. In what follows, we have denoted vectors and matrices using bold-faced lower-case and upper-case characters, respectively, and vectors are always in column form.

\section{Two-Channel LMS/Newton ALGORITHM}

In [7], we presented two versions of the LMS/Newton algorithm based on AR modeling for the stereophonic signals. Modeling the speech input signals to the adaptive filters as AR processes of order $M$, where $M \ll L$, results in an efficient way of updating $\mathbf{R}_{x x}^{-1} \mathbf{x}(n)$ (see the update (6) below) without having to estimate the input autocorrelation matrix $\mathbf{R}_{x x}=E\left[\mathbf{x}(n) \mathbf{x}(n)^{T}\right]$, where $E[\cdot]$ denotes expectation and $\mathbf{x}(n)$ is the $2 L \times 1$ filter input vector given as $\mathbf{x}(n)=\left[x_{1}(n), x_{2}(n), x_{1}(n-1), x_{2}(n-1), \ldots, x_{1}(n-L+\right.$ $\left.1), x_{2}(n-L+1)\right]^{T}$. These low complexity adaptive algorithms make use of the properties of the two-channel gradient lattice algorithm [7], [10], where the backward prediction-errors of $\mathbf{x}(n)$, denoted by $\mathbf{b}(n)$ can be expressed as

$$
\mathbf{b}(n)=\mathbf{L x}(n) .
$$

In (3) [see (4), shown at the bottom of the page], where $\mathbf{I}_{2}$ is a $2 \times 2$ identity matrix, $\mathbf{0}$ is a $2 \times 2$ zero matrix and each $\mathbf{G}_{m, j}$ is a $2 \times 2$ backward predictor coefficient matrix [9]. Equation (3) is also known as the Gram-Schmidt orthogonalization algorithm [11]. This algorithm provides a one-to-one correspondence between the input vector $\mathbf{x}(n)$ and the backward prediction-error vector $\mathbf{b}(n)$. From (3), it follows that

$$
\mathbf{R}_{x x}^{-1}=\mathbf{L}^{T} \mathbf{R}_{b b}^{-1} \mathbf{L}
$$

where $\mathbf{R}_{b b}=E\left[\mathbf{b}(n) \mathbf{b}^{T}(n)\right]$. The update equation for the ideal LMS/Newton algorithm is [7], [9]

$$
\hat{\mathbf{h}}(n+1)=\hat{\mathbf{h}}(n)+2 \mu \mathbf{R}_{x x}^{-1} \mathbf{x}(n) e(n)
$$

where $\mu$ is a small step-size used to control the adaptation and $\hat{\mathbf{h}}(n)=\left[\hat{h}_{1,0}(n), \hat{h}_{2,0}(n), \hat{h}_{1,1}(n), \hat{h}_{2,1}(n), \ldots\right.$, $\left.\hat{h}_{1, L-1}(n), \hat{h}_{2, L-1}(n)\right]^{T}$. We note that the filter coefficients of

$$
\mathbf{L}=\left[\begin{array}{cccccc}
\mathbf{I}_{2} & \mathbf{0} & \mathbf{0} & \cdots & \mathbf{0} & \mathbf{0} \\
-\mathbf{G}_{1,1} & \mathbf{I}_{2} & \mathbf{0} & \cdots & \mathbf{0} & \mathbf{0} \\
-\mathbf{G}_{2,1} & -\mathbf{G}_{2,2} & \mathbf{I}_{2} & \cdots & \mathbf{0} & \mathbf{0} \\
\vdots & \vdots & \vdots & \ddots & \vdots & \vdots \\
-\mathbf{G}_{L-1,1} & -\mathbf{G}_{L-1,2} & -\mathbf{G}_{L-1,3} & \cdots & -\mathbf{G}_{L-1, L-1} & \mathbf{I}_{2}
\end{array}\right]
$$




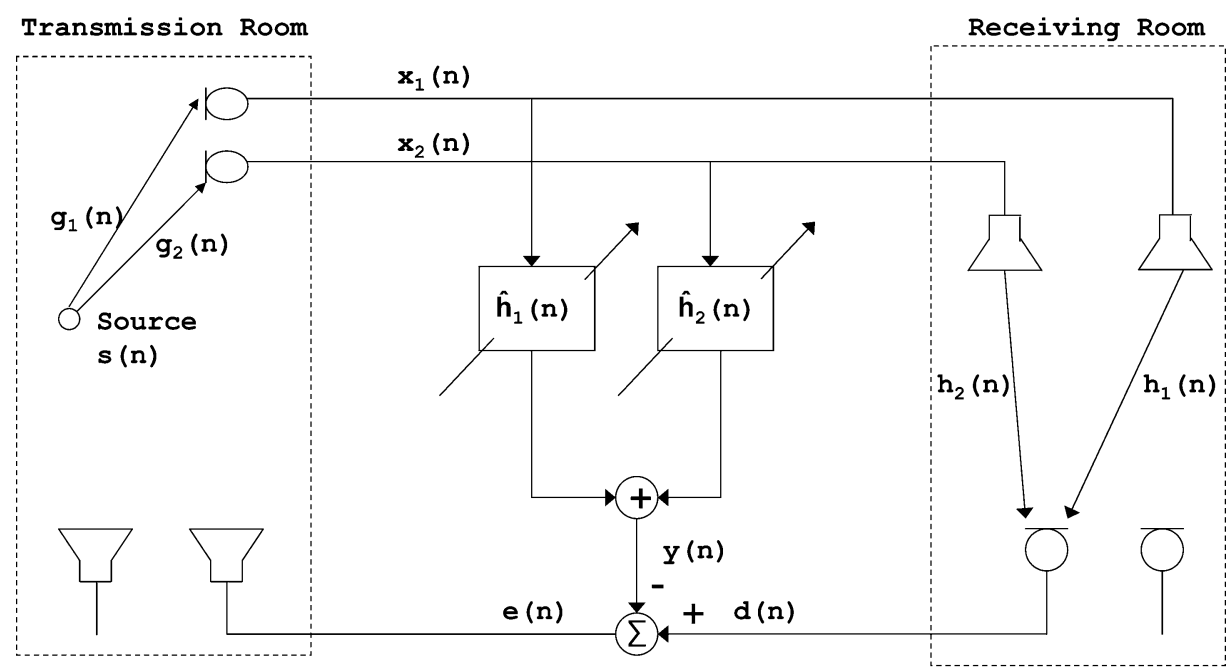

Fig. 1. Setup of a stereophonic acoustic echo cancellation system.

the two channels are interleaved to form $\hat{\mathbf{h}}(n)$ in order to be consistent with the above definition of the input vector $\mathbf{x}(n)$. Using (3) and (5), (6) can be written as

$$
\hat{\mathbf{h}}(n+1)=\hat{\mathbf{h}}(n)+\mu \mathbf{u}(n) e(n)
$$

where

$$
\mathbf{u}(n)=\mathbf{L}^{T} \mathbf{R}_{b b}^{-1} \mathbf{b}(n) .
$$

The first version of the two-channel LMS/Newton algorithm (referred to as Algorithm 1 in [7]) will involve the direct implementation of (8) through the use of a two-channel lattice predictor. Since we are assuming the input sequence $\mathbf{x}(n)$ to be an AR process of order $M \ll L$, a lattice predictor of order $M$ is sufficient. The matrix $\mathbf{L}$ then takes the form shown in (9) at the bottom of the page, and the vector $\mathbf{b}(n)$ takes the form of

$$
\begin{aligned}
\mathbf{b}(n)= & {\left[b_{1,0}(n), b_{2,0}(n), b_{1,1}(n), b_{2,1}(n), \ldots, b_{1, M}(n),\right.} \\
& b_{2, M}(n), b_{1, M}(n-1), b_{2, M}(n-1), \ldots, \\
& \left.b_{1, M}(n-L+M+1), b_{2, M}(n-L+M+1)\right]^{T} .
\end{aligned}
$$

A close examination of the structures of the $\mathbf{L}$ matrix and $\mathbf{b}(n)$ vector described in (9) and (10), respectively, will reveal that in order to compute $\mathbf{u}(n)$, only the first $2(M+1)$ and the last $2 M$ elements of $\mathbf{u}(n)$ need to be computed. The remaining elements of $\mathbf{u}(n)$ are the delayed versions of its $(2 M+1)$ th and $(2 M+2)$ nd elements. A detailed exposition of the implemen- tation of the algorithm can be found in [7]. Despite having reasonably low computational complexity, this particular version of the two-channel LMS/Newton algorithm is structurally complicated as it involves extensive ordering of manipulated data. While the implementation of the Algorithm 1 can be easily done using software, it may be difficult to do the same on a custom chip using very-large-scale integration (VLSI).

In [7], we have also derived an alternate Algorithm 2 that is computationally less complex and can be easily implemented in hardware. While Algorithm 1 will be an exact implementation of the ideal two-channel LMS/Newton algorithm, an approximation was introduced in evaluating (8) in Algorithm 2. Similar to the set of backward predictor coefficient matrices $\mathbf{G}_{m, j}$, there exists a corresponding set of $2 \times 2$ forward predictor coefficient matrices denoted by $\mathbf{A}_{m, j}$. It is well known that in a single-channel lattice $g_{m, j}=a_{m, m+1-j}, j=1,2, \ldots, m$ and this relationship between the forward and backward predictor coefficients was used to derive the single-channel LMS/Newton algorithm in [8]. In a two-channel lattice, $\mathbf{G}_{m, j}=\mathbf{A}_{m, m+1-j}^{T}$ for only $j=1$ [7]. But based on the perspective gained from extensive experimentation, we observed that this relationship also approximately holds true for $j=2,3, \ldots, m$. Hence, we introduced the approximate relationship between the forward and backward predictor coefficients as $\mathbf{G}_{m, j}=\mathbf{A}_{m, m+1-j}^{T}, j=$ $1,2, \ldots, m$. This approximation was used to derive an algorithm having much lower complexity without compromising the performance significantly. The details of this particular algorithm can also be found in [7].

$$
\mathbf{L}=\left[\begin{array}{cccccccccc}
\mathbf{I}_{2} & \mathbf{0} & \cdots & \mathbf{0} & \mathbf{0} & \cdots & \mathbf{0} & \mathbf{0} & \cdots & \mathbf{0} \\
-\mathbf{G}_{1,1} & \mathbf{I}_{2} & \cdots & \mathbf{0} & \mathbf{0} & \cdots & \mathbf{0} & \mathbf{0} & \cdots & \mathbf{0} \\
\vdots & \vdots & \ddots & \vdots & \vdots & \ddots & \vdots & \vdots & \ddots & \vdots \\
-\mathbf{G}_{M, 1} & -\mathbf{G}_{M, 2} & \cdots & \mathbf{I}_{2} & \mathbf{0} & \cdots & \mathbf{0} & \mathbf{0} & \cdots & \mathbf{0} \\
\mathbf{0} & -\mathbf{G}_{M, 1} & \cdots & -\mathbf{G}_{M, M} & \mathbf{I}_{2} & \cdots & \mathbf{0} & \mathbf{0} & \cdots & \mathbf{0} \\
\vdots & \vdots & \ddots & \vdots & \vdots & \ddots & \vdots & \vdots & \ddots & \vdots \\
\mathbf{0} & \mathbf{0} & \cdots & \mathbf{0} & \mathbf{0} & \cdots & -\mathbf{G}_{M, 1} & -\mathbf{G}_{M, 2} & \cdots & \mathbf{I}_{2}
\end{array}\right]
$$




\section{ANALYSIS OF TWO-CHANNEL LMS/NEWTON ALGORITHM}

In this section, we present an analysis of the two-channel LMS/Newton algorithm. Before we proceed with the derivations, we provide a description of the models used to generate the two-channel input signals. In this work, the room echo paths are modeled as finite-impulse response (FIR) filters and are chosen to be independent, zero-mean Gaussian sequences, each having a variance that decays at the rate of $(1 / n)$, where $n$ is the sample number. The length of the adaptive filters modeling the acoustic echo cancelers, the length of the near-end room echo paths, and the length of the far-end room echo paths will be denoted by $L, L_{N}$, and $L_{F}$, respectively. The reference input $x_{1}(n)$ to the one of the adaptive filters is generated by filtering a zero-mean, unit variance Gaussian sequence through a far-end room echo path. We generate the other reference input $x_{2}(n)$ using the following relation [12]:

$$
x_{2}(n)=\sqrt{\gamma} x_{1}(n)+\sqrt{1-\gamma} b_{w}(n)
$$

where $b_{w}(n)$ is a zero-mean, unit variance Gaussian random variable and we have used $\gamma=0.99$ to reflect the high interchannel correlation that may exist between the two-channel input signals.

First, we present an analysis of the convergence of $\hat{\mathbf{h}}(n)$ in the mean. Next, we proceed to analyze the mean-square convergence of $\hat{\mathbf{h}}(n)$, which will give an equation for the misadjustment of the algorithm. We assume the input samples $x_{1}(n), x_{2}(n)$ and the desired output samples $d(n)$ consist of mutually Gaussian-distributed random variables, for all $n$. Recall, previously, the two-channel vectors were formed by interleaving the corresponding two-channel elements. This was necessary to derive the algorithms in [7]. However, to make the analysis convenient, henceforth, the various two-channel elements are no longer interleaved to form the corresponding two-channel vector. For example, $\mathbf{x}(n)$ is now defined as $\mathbf{x}(n)=\left[x_{1}(n), x_{1}(n-\right.$ $\left.1), \ldots, x_{1}(n-L+1), x_{2}(n), x_{2}(n-1), \ldots, x_{2}(n-L+1)\right]^{T}$ and $\hat{\mathbf{h}}(n)$ is defined as $\hat{\mathbf{h}}(n)=\left[\hat{h}_{1,0}(n), \hat{h}_{1,1}(n), \ldots\right.$, $\left.\hat{h}_{1, L-1}(n), \hat{h}_{2,0}(n), \hat{h}_{2,1}(n), \ldots, \hat{h}_{2, L-1}(n)\right]^{T}$. As will become evident, these slight misnomer of notations are important and greatly simply our presentation.

\section{A. Mean Analysis of Tap-Weight Vector}

We will examine the convergence of $E[\hat{\mathbf{h}}(n)]$ as $n$ increases. If we define a coefficient error vector as $\mathbf{v}(n)=\hat{\mathbf{h}}(n)-\mathbf{h}_{o}$, where $\mathbf{h}_{o}$ denotes the optimal filter coefficients, we note that

$$
e(n)=d(n)-\mathbf{x}^{T}(n) \hat{\mathbf{h}}(n)=e_{o}(n)-\mathbf{x}^{T}(n) \mathbf{v}(n)
$$

where

$$
e_{o}(n)=d(n)-\mathbf{x}^{T}(n) \mathbf{h}_{o}
$$

According to the principle of orthogonality, we have $E\left[e_{o}(n) \mathbf{x}(n)\right]=0$ [9], [11]. Then, it follows from (3), $(7),(8)$, and (12) that

$$
E[\mathbf{v}(n+1)]=\left[\mathbf{I}_{2 L}-2 \mu E\left[\mathbf{u}(n) \mathbf{x}^{T}(n)\right]\right] E[\mathbf{v}(n)]
$$

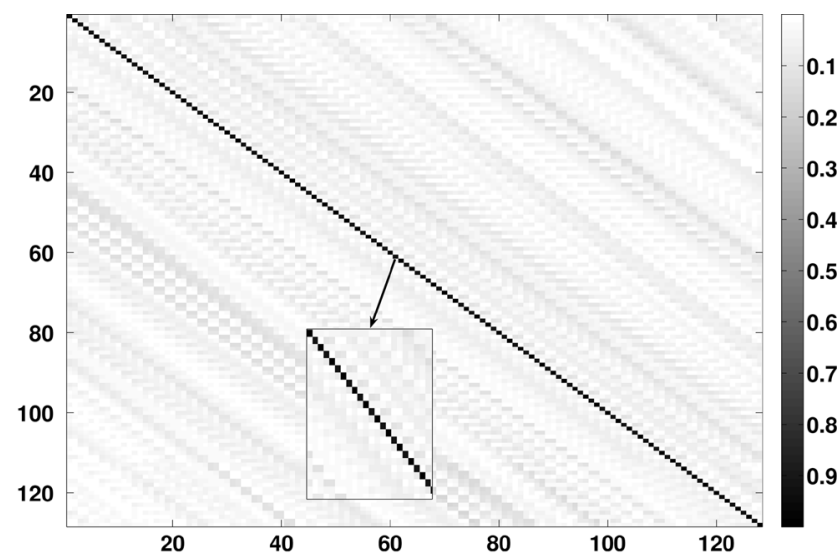

(a)

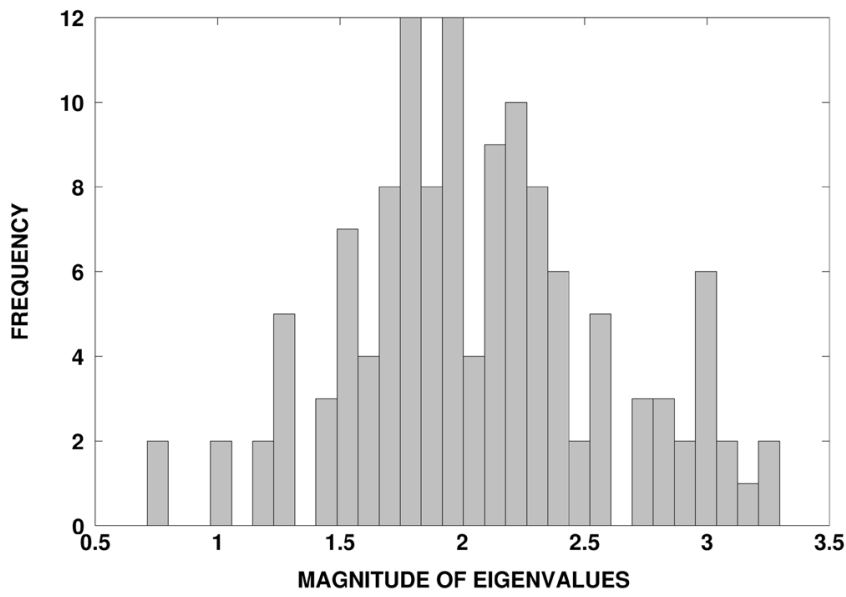

(b)

Fig. 2. (a) Covariance matrix $\mathbf{R}_{u x}=E\left[\mathbf{u}(n) \mathbf{x}^{T}(n)\right]$ for Algorithm 1 . (b) Histogram of the eigenvalues of $\mathbf{R}_{u x}$.

where $\mathbf{I}_{2 L}$ is a $2 L \times 2 L$ identity matrix. If we let $\mathbf{R}_{u x}=$ $E\left[\mathbf{u}(n) \mathbf{x}^{T}(n)\right]$, we observe that the convergence of Algorithm 1 is controlled by the eigenvalues of the matrix $\mathbf{R}_{u x}$. From (3), (5), and (8), we know that, ideally, $\mathbf{R}_{u x}=\mathbf{I}_{2 L}$. Simulations are performed to confirm the diagonal nature of $\mathbf{R}_{u x}$. We chose $L_{F}=128$ and $L=L_{N}=64$ and results are obtained by averaging over 10 independent runs.

Fig. 2(a) shows a plot of the magnitude of the elements of $\mathbf{R}_{u x}$ and Fig. 2(b) shows the histogram of the corresponding eigenvalues of $\mathbf{R}_{u x}$. This experiment clearly shows that the Algorithm 1 will not suffer from any significant eigenvalue spread and its behavior will be governed by nearly equal modes of convergence.

A careful examination of the $\mathbf{R}_{u x}$ matrix for Algorithm 2 as shown in Fig. 3(a) will reveal that the matrix is banded and it is only approximately diagonal. This is to be expected because we had introduced an approximation in the derivation of Algorithm 2 in [7]. However, as depicted in Fig. 3(b), this particular version of the LMS/Newton algorithm is still able to achieve a relatively small eigenvalue spread. Hence, this algorithm while being computationally more efficient, will only converge slightly slower than Algorithm 1. This simulation example provides further validation of our results presented in [7]. 


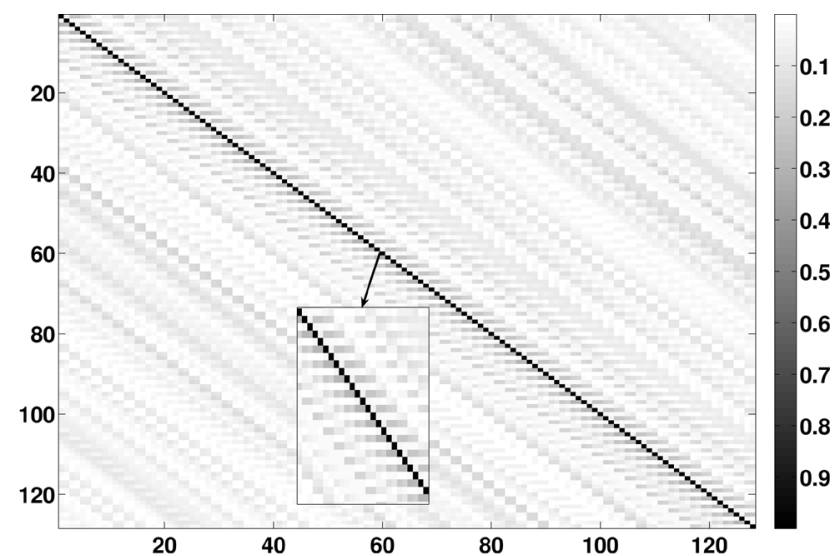

(a)

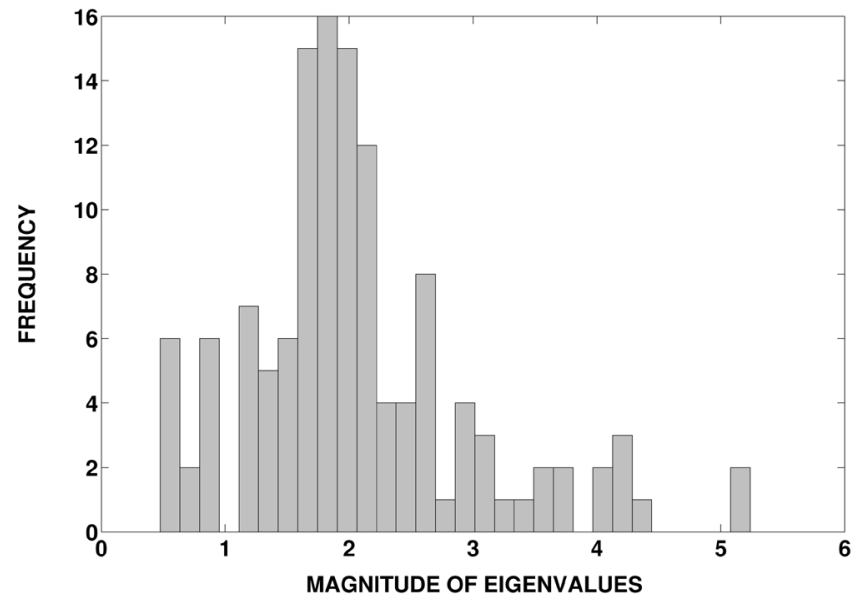

(b)

Fig. 3. (a) Covariance matrix $\mathbf{R}_{u x}=E\left[\mathbf{u}(n) \mathbf{x}^{T}(n)\right]$ for Algorithm 2 . (b) Histogram of the eigenvalues of $\mathbf{R}_{u x}$.

\section{B. Mean-Square Analysis of Tap-Weight Vector}

We now proceed to present a mean-square analysis of the tapweight vector for the LMS/Newton Algorithm 1. The banded nature of $\mathbf{R}_{u x}$ will make the task of providing a mathematical analysis for Algorithm 2 more complicated. Hence, we will restrict ourselves to the analysis of Algorithm 1.

We first define the correlation matrix of the tap-weight error vector $\mathbf{v}(\mathrm{n})$ as $\mathbf{K}(n)=E\left[\mathbf{v}(n) \mathbf{v}^{T}(n)\right]$ and then examine its evolution over time. To this end, we take the outer product of both sides of (14) and take the expectations of the left- and rightsides of the resulting equation. Thus, we obtain

$$
\begin{aligned}
\mathbf{K}(n+1)=E\left[\left[\mathbf{I}_{2 L}-2 \mu \mathbf{u}(n) \mathbf{x}^{T}(n)\right] \cdot \mathbf{K}(n)\right. \\
\left.\cdot\left[\mathbf{I}_{2 L}-2 \mu \mathbf{x}(n) \mathbf{u}^{T}(n)\right]\right]+4 \mu^{2} J_{\min } \mathbf{R}_{u u}
\end{aligned}
$$

where $J_{\min }=E\left[e_{o}^{2}(n)\right]$ and $\mathbf{R}_{u u}=E\left[\mathbf{u}(n) \mathbf{u}^{T}(n)\right]$. This results in

$$
\begin{aligned}
\mathbf{K}(n+1)= & \mathbf{K}(n)-2 \mu E\left[\mathbf{u}(n) \mathbf{x}^{T}(n)\right] \mathbf{K}(n) \\
& -2 \mu E\left[\mathbf{x}(n) \mathbf{u}^{T}(n)\right] \mathbf{K}(n)+4 \mu^{2} J_{\min } \mathbf{R}_{u u} \\
& +4 \mu^{2} E\left[\mathbf{u}(n) \mathbf{x}^{T}(n) \mathbf{K}(n) \mathbf{x}(n) \mathbf{u}^{T}(n)\right] .
\end{aligned}
$$

Before we proceed further, we define $\mathbf{K}(n)$ as

$$
\mathbf{K}(n)=\left[\begin{array}{ll}
\mathbf{K}_{11}(n) & \mathbf{K}_{12}(n) \\
\mathbf{K}_{21}(n) & \mathbf{K}_{22}(n)
\end{array}\right]
$$

where each $\mathbf{K}_{i j}(n)$ is a matrix of dimension $L \times L$ and $i, j=$ 1,2 represent the channel indices. Similarly, $\mathbf{R}_{x x}$ can take the form of

$$
\mathbf{R}_{x x}=\left[\begin{array}{ll}
\mathbf{R}_{x_{1} x_{1}} & \mathbf{R}_{x_{1} x_{2}} \\
\mathbf{R}_{x_{2} x_{1}} & \mathbf{R}_{x_{2} x_{2}}
\end{array}\right] .
$$

We further define $\Delta \mathbf{K}(n)=\mathbf{K}_{12}(n)-\mathbf{K}_{21}(n)$ and $\Delta \mathbf{R}_{x x}=$ $\mathbf{R}_{x_{1} x_{2}}-\mathbf{R}_{x_{2} x_{1}}$.

An evaluation of the term $E\left[\mathbf{u}(n) \mathbf{x}^{T}(n) \mathbf{K}(n) \mathbf{x}(n) \mathbf{u}^{T}(n)\right]$ as provided in Appendix A, gives

$$
\begin{aligned}
& E\left[\mathbf{u}(n) \mathbf{x}^{T}(n) \mathbf{K}(n) \mathbf{x}(n) \mathbf{u}^{T}(n)\right] \\
& =\operatorname{tr}\left[\mathbf{K}(n) \mathbf{R}_{x x}\right] \mathbf{R}_{u u}+\operatorname{tr}\left[\Delta \mathbf{K}(n) \Delta \mathbf{R}_{x x}\right] \mathbf{R}_{u u}+2 \mathbf{K}(n)
\end{aligned}
$$

where $\operatorname{tr}[\cdot]$ denotes the sum of the diagonal elements of the corresponding matrix.

Using this result in (16), we obtain

$$
\begin{aligned}
\mathbf{K}(n+1)= & \mathbf{K}(n)-2 \mu \mathbf{R}_{u x} \mathbf{K}(n)-2 \mu \mathbf{K}(n) \mathbf{R}_{u x} \\
& +4 \mu^{2} \operatorname{tr}\left[\mathbf{K}(n) \mathbf{R}_{x x}\right] \mathbf{R}_{u u}+4 \mu^{2} \operatorname{tr}\left[\Delta \mathbf{K}(n) \Delta \mathbf{R}_{x x}\right] \\
& \cdot \mathbf{R}_{u u}+8 \mu^{2} \mathbf{K}(n)+4 \mu^{2} J_{\min } \mathbf{R}_{u u} .
\end{aligned}
$$

We note that the excess mean-square error (MSE) of an adaptive filter with an input correlation matrix $\mathbf{R}_{x x}$ and a tap-weight error correlation matrix $\mathbf{K}(n)$ for a single-channel case is given by $J_{e x}(n)=\operatorname{tr}\left[\mathbf{K}(n) \mathbf{R}_{x x}\right]$ [9]. It is trivial to show that this expression is valid for the two-channel case as well. Also, using (5) and (8), we know that $\mathbf{R}_{u u}=\mathbf{R}_{x x}^{-1}$. Hence, we have $\operatorname{tr}\left[\mathbf{R}_{u u} \mathbf{R}_{x x}\right]=2 L$.

We obtain the recursive equation for the time evolution of the excess MSE by multiplying both sides of (20) by $\mathbf{R}_{x x}$ and taking the traces of the resulting equation. This gives

$$
\begin{aligned}
J_{e x}(n+1)= & J_{e x}(n)-2 \mu \operatorname{tr}\left[\mathbf{R}_{u x} \mathbf{K}(n) \mathbf{R}_{x x}\right] \\
& -2 \mu \operatorname{tr}\left[\mathbf{K}(n) \mathbf{R}_{u x} \mathbf{R}_{x x}\right]+8 \mu^{2} L J_{e x}(n) \\
& +8 \mu^{2} L \operatorname{tr}\left[\Delta \mathbf{K}(n) \Delta \mathbf{R}_{x x}\right]+8 \mu^{2} J_{e x}(n) \\
& +8 \mu^{2} L J_{\min } .
\end{aligned}
$$

Rearranging the terms results in

$$
\begin{aligned}
J_{e x}(n+1)= & {\left[1+8 \mu^{2} L+8 \mu^{2}\right] J_{e x}(n) } \\
& -2 \mu \operatorname{tr}\left[\mathbf{R}_{u x} \mathbf{K}(n) \mathbf{R}_{x x}\right]-2 \mu \operatorname{tr}\left[\mathbf{R}_{u x} \mathbf{R}_{x x} \mathbf{K}(n)\right] \\
& +8 \mu^{2} L \operatorname{tr}\left[\Delta \mathbf{K}(n) \Delta \mathbf{R}_{x x}\right]+8 \mu^{2} L J_{\min } .
\end{aligned}
$$

We note that $\operatorname{tr}\left[\mathbf{R}_{u x}(n) \mathbf{K}(n) \mathbf{R}_{x x}\right]=\operatorname{tr}\left[\mathbf{R}_{u x} \mathbf{R}_{x x} \mathbf{K}(n)\right] \approx$ $\operatorname{tr}\left[\mathbf{R}_{x x} \mathbf{K}(n)\right]=J_{e x}(n)$, since the simulation results depicted in Fig. 2 indicate that $\mathbf{R}_{u x} \approx \mathbf{I}_{2 L}$.

Equation (22) can now be written as

$$
\begin{aligned}
& J_{e x}(n+1)=\left[1-4 \mu+8 \mu^{2} L+8 \mu^{2}\right] J_{e x}(n) \\
&+8 \mu^{2} L \operatorname{tr}\left[\Delta \mathbf{K}(n) \Delta \mathbf{R}_{x x}\right]+8 \mu^{2} L J_{\min } .
\end{aligned}
$$


TABLE I

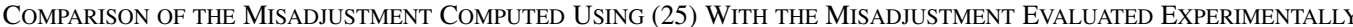

\begin{tabular}{|c|c|c|}
\hline CORRELATION COEFFICIENT $(\gamma)$ & MISADJUSTMENT (THEORETICAL) & MISADJUSTMENT (EXPERIMENTAL) \\
\hline 0.0 & 0.1110 & 0.1121 \\
0.1 & 0.1112 & 0.1128 \\
0.5 & 0.1114 & 0.1120 \\
0.9 & 0.1114 & 0.1130 \\
0.99 & 0.1121 & 0.1197 \\
\hline
\end{tabular}

From (23), we note that the convergence of the algorithm is guaranteed if $\left[1-4 \mu+8 \mu^{2} L+8 \mu^{2}\right]<1$. This gives

$$
0<\mu<\frac{1}{2 L+2} \text {. }
$$

Furthermore, when $n \rightarrow \infty$, we can use (23) to derive an expression for the misadjustment of Algorithm 1 as [9], [11]

$$
\mathcal{M}=\frac{J_{e x}(\infty)}{J_{\min }}=\lim _{n \rightarrow \infty} \frac{2 \mu L\left[\frac{\operatorname{tr}\left[\Delta \mathbf{K}(n) \Delta \mathbf{R}_{x x}\right]}{J_{\min }}+1\right]}{1-2 \mu(L+1)} .
$$

For the two-channel case, $\mathbf{R}_{x_{1} x_{2}} \neq \mathbf{R}_{x_{2} x_{1}}$, and hence, $\Delta \mathbf{R}_{x x} \neq$ 0 . However, when the two-channel input signals are uncorrelated, $\Delta \mathbf{R}_{x x}=0$ and the misadjustment as given by (25) reduces to those of the single-channel LMS/Newton Algorithm 1 with a tap-weight vector of length $2 L$ [8].

We note that it is not possible to obtain the misadjustment in practical situations due to the inability to measure $\mathbf{K}(n)$. However, the accuracy of (25) can be verified using simulations. As done previously in Section III-A, we chose $L_{F}=128$ and $L=L_{N}=64$ and results are obtained by averaging over 10 independent runs. Table I compares the theoretical misadjustment obtained using (25) with the empirically determined values of misadjustment for different levels of correlation between the two-channel input signals. We observe that there is a slight difference between the theoretical misadjustment and the experimentally measured misadjustment. This is to be expected because we made an assumption that $\mathbf{R}_{u x}$ is an identity matrix in deriving (25) whereas simulations results showed us that $\mathbf{R}_{u x}$ is only nearly identical. Furthermore, we note that similar small numerical differences between theoretical and empirical values have also been observed in the past in the theory of LMS algorithms [9], [11].

\section{IMPACT OF NONLINEARITY ON THE CONVERGENCE BEHAVIOR}

The nonlinearity technique proposed in [4] has been shown to be very effective in improving the misalignment problem. The incorporation of a nonlinear preprocessor will reduce the interchannel correlation and improve the conditioning of the autocorrelation matrix $\mathbf{R}_{x x}$, thereby reducing the misalignment obtained by the adaptive algorithms [4]. For highly correlated but non-singular, simulated stereophonic AEC systems, the twochannel LMS/Newton algorithms developed in [7] were able to converge faster in terms of the MSE and also achieve lower misalignment than the other state-of-the-art algorithms of comparable complexity [7]. Simulation studies showed that the twochannel LMS/Newton algorithm will benefit from the use of nonlinearities by exhibiting a further improvement in the misalignment. However, it was also observed that certain types of nonlinearities introduce slow modes of convergence in the MSE. This would appear contradictory to the results of our discussion in Section III-A, wherein we showed that the two-channel LMS/Newton algorithm will exhibit nearly equal modes of convergence, irrespective of the nature of the input signal. In the rest of the paper, we will discuss the impact of nonlinearities on the convergence behavior of the two-channel LMS/Newton algorithm, in particular Algorithm 1.

Of the several types of nonlinearities evaluated in [13], a halfwave rectifier has been subjectively shown to perform well for speech signals. A modified version of this transformation that uses a positive half-wave rectifier for one channel and a negative half-wave rectifier for the other channel is commonly employed in the literature [5], [10], [13]. Over here, the two-channel stereo input signals $x_{1}(n)$ and $x_{2}(n)$ to the adaptive filters are preprocessed as shown below [5]:

$$
\begin{aligned}
& \tilde{x}_{1}(n)=x_{1}(n)+0.5 \alpha\left(x_{1}(n)+\left|x_{1}(n)\right|\right) \\
& \tilde{x}_{2}(n)=x_{2}(n)+0.5 \alpha\left(x_{2}(n)-\left|x_{2}(n)\right|\right)
\end{aligned}
$$

where $\alpha$ is used to control the level of nonlinear distortion.

A hard limiter can also be used to distort the two-channel input signals as follows [5]:

$$
\tilde{x}_{i}(n)=x_{i}(n)+\alpha \operatorname{sgn}\left(x_{i}(n)\right) ; \quad i=1,2
$$

where $\operatorname{sgn}(x)=(|x|) /(x)$. We will use both the above transformations to analyze the impact of nonlinearities on the convergence of the two-channel LMS/Newton algorithm.

We will demonstrate the performance of the two-channel LMS/Newton adaptive algorithm in the presence of the above nonlinearities by a simulation example. In particular, we would like to bring forth the effect of nonlinearities on the convergence rate of the MSE of the LMS/Newton Algorithm 1. The exclusive tap-selection criterion has been shown to improve the convergence rate of several adaptive algorithms [5]. The XM selective adaptation technique can be applied to the NLMS algorithm and this implementation has a complexity comparable to our algorithm. Hence, we have compared the performance of the LMS/Newton Algorithm 1 with the XM-NLMS algorithm of [5].

In this simulation, the length of the modeling adaptive filters $L$ is set equal to 64 and the length of the near-end room echo paths $L_{N}$ is also selected to be the same. The length of the far-end room echo path $L_{F}$ is equal to 128 . We chose $\alpha=0.5$ as this provides a satisfactory trade-off between quality of stereo perception and reduction of misalignment [4]. Upon extensive experimentation, it was observed that the LMS/Newton Algorithm 1 exhibited satisfactory performance when the order of 


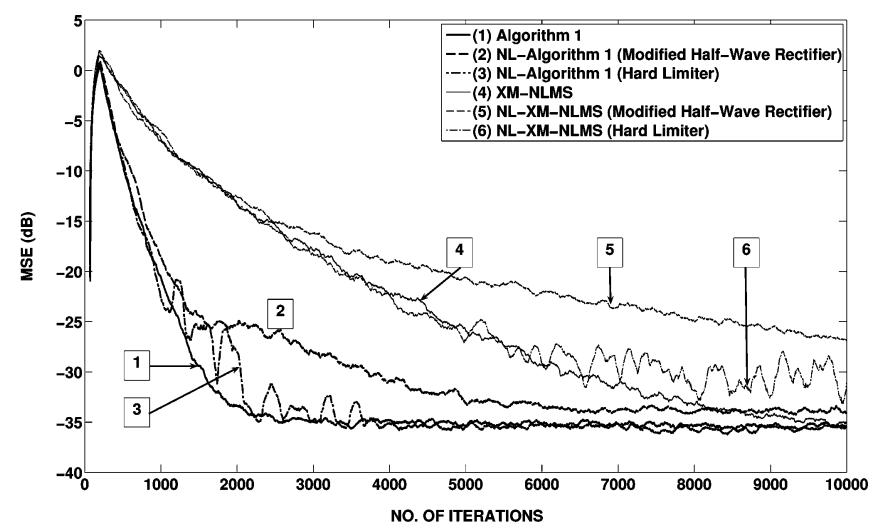

(a)

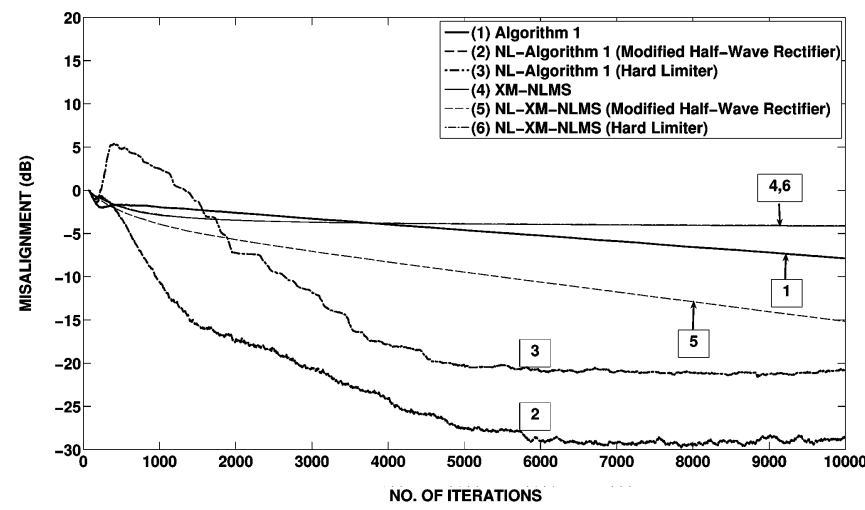

(b)

Fig. 4. (a) Comparison of the MSE. (b) Comparison of the misalignment (Colored Gaussian noise inputs, $L=64$, Thick solid-Algorithm 1, Thick dashed-Algorithm 1 with nonlinearity (26), Thick dashed-dotted-Algorithm 1 with nonlinearity (27), Thin solid-XM-NLMS, Thin dashed-XM-NLMS with nonlinearity (26), Thin dashed-dotted-XM-NLMS with nonlinearity (27).)

the AR model $M$ is chosen to be 8 . For the XM-NLMS algorithm, we chose the size of the tap-selection set (denoted by the parameter $M$ in [5]) to be $0.5 L$. Uncorrelated noise is added to $d(n)$ such that a SNR of $40 \mathrm{~dB}$ is achieved and the simulation results are obtained after averaging over 10 independent runs. The measures of the MSE and the normalized misalignment are chosen as metrics for performance evaluation. Fig. 4(a) compares the MSE and Fig. 4(b) compares the normalized misalignment curves of the LMS/Newton Algorithm 1 and the XM-NLMS algorithm with the cases when the two nonlinearities described above in (26) and (27) are introduced.

When we do not add nonlinearity to the adaptive algorithms, we can see that the LMS/Newton Algorithm 1 does not suffer from the eigenvalue spread problem and has nearly equal modes of convergence. The use of nonlinearities also results in a significant improvement in the misalignment obtained by both the adaptive algorithms, except for the case when a hard limiter is incorporated with the XM-NLMS algorithm. ${ }^{1}$ However, as is apparent from Fig. 4(a), the more significant outcome of adding nonlinearities is the appearance of the slow modes of convergence when the modified half-wave rectifier is used in conjunction with the LMS/Newton Algorithm 1. This is surprising given that the LMS/Newton Algorithm 1 should have nearly equal

${ }^{1}$ This particular transformation has also been subjectively shown to perform poorly for speech signals [13].

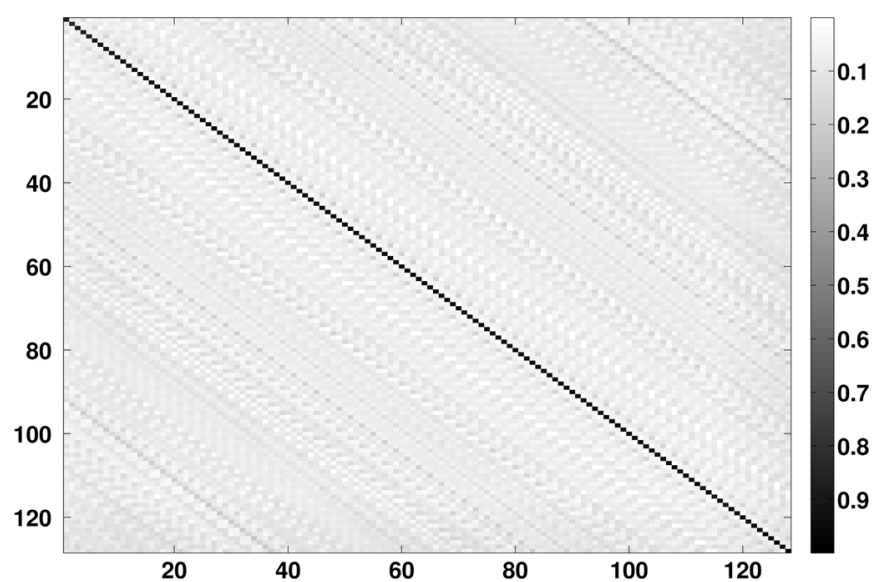

(a)

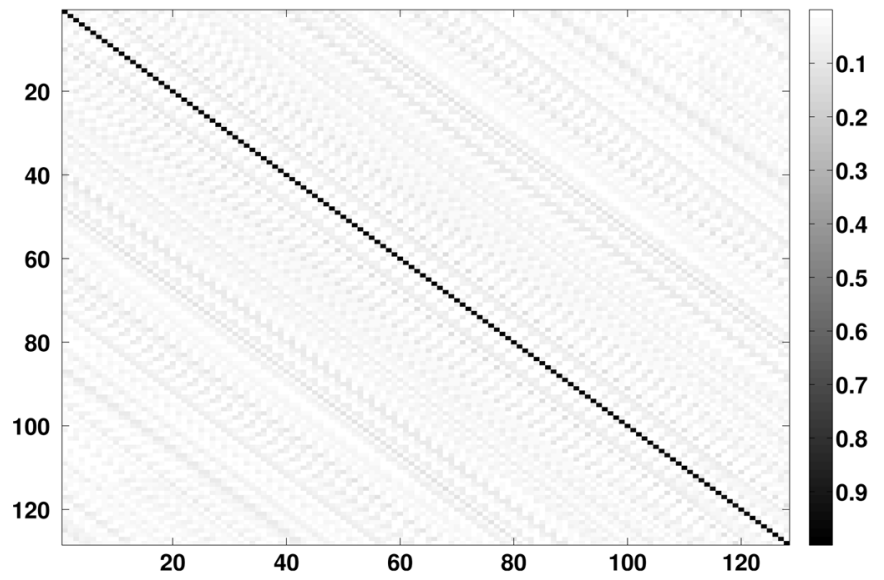

(b)

Fig. 5. Covariance matrix $\mathbf{R}_{u x}$ for Algorithm 1. (a) A modified half-wave rectifier is used. (b) A hard limiter is used.

TABLE II

CONDITION Numbers OF $\mathbf{R}_{u x}$ FOR LMS/NEWTON ALGORITHM 1

\begin{tabular}{|c|c|}
\hline TYPE OF NONLINEARITY & $\chi\left(\mathbf{R}_{u x}\right)$ \\
\hline No nonlinearity & 7.4821 \\
Modified half-wave rectifier & 50.0659 \\
Hard limiter & 6.9060 \\
\hline
\end{tabular}

modes of convergence, irrespective of the nature of the input signal due to the presence of the normalization matrix $\mathbf{R}_{x x}^{-1}$ in (6) [7].

Investigating further, we know from (14), the eigenvalues of the covariance matrix $\mathbf{R}_{u x}$ controls the convergence of Algorithm 1. We also observed earlier in Fig. 2, that in the absence of nonlinearities, $\mathbf{R}_{u x}$ is close to the identity matrix. However, with the introduction of nonlinearities, a careful examination of $\mathbf{R}_{u x}$ will provide more insight into understanding this strange behavior of the LMS/Newton Algorithm 1. In Fig. 5, we present pictorial representations of the covariance matrix $\mathbf{R}_{u x}$ for the cases when (a) a modified half-wave rectifier is used; (b) a hard limiter is used. These plots clearly indicate that the contribution of the non-diagonal elements of $\mathbf{R}_{u x}$ in much greater when a modified half-wave rectifier is used as compared to the case when a hard limiter is used. Consequently, $\mathbf{R}_{u x}$ has a larger eigenvalue spread and this is reflected in the condition numbers listed in Table II. Thus, using a half-wave rectification results in 


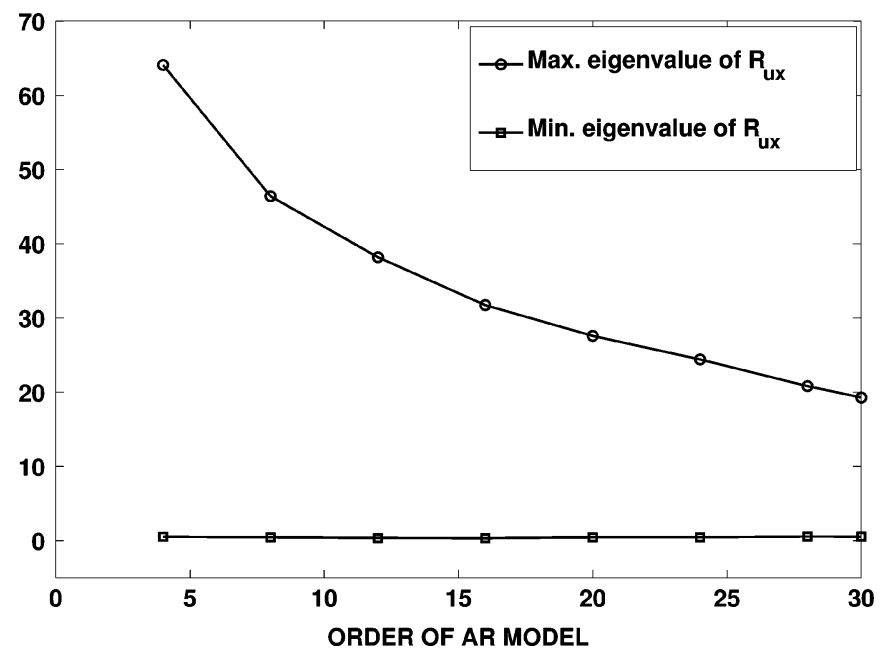

Fig. 6. Variation of the maximum and minimum eigenvalues of $\mathbf{R}_{u x}$.

the poor conditioning of $\mathbf{R}_{u x}$, which will explain the presence of the slow modes in the learning curve as depicted in Fig. 4(a).

This leads to the question of why only the nonlinearity from (26) and not (27) degrades the conditioning of $\mathbf{R}_{u x}$. Recall that the computation of $\mathbf{u}(n)$ involves the use of a two-channel lattice predictor. Furthermore, we assume the input signals $x_{1}(n)$ and $x_{2}(n)$ to be AR processes of order $M$, where $M \ll L$. We note that the key difference between the two nonlinearities as described in (26) and (27) is the presence of a strong direct current $(\mathrm{dc})$ bias in the case of a half-wave rectifier, which is altogether absent in the case of a hard limiter. Intuitively, this would suggest a limitation of the AR model based computation of $\mathbf{R}_{x x}^{-1}$, according to (5), using a two-channel lattice predictor in the presence of dc components.

We computed the magnitude of the maximum and minimum eigenvalues of $\mathbf{R}_{u x}$ for various orders of the AR model $M$ and for a fixed length of the modeling adaptive filters $L=64$ and this is depicted in Fig. 6. The results were obtained when the two-channel stereo input signals were transformed using the modified half-wave rectifier. The plot indicates that the accuracy of the AR model based computation of $\mathbf{u}(n)$ in the presence of a dc bias improves with an increase in the order of the AR model. As a result, there is a corresponding decrease in the eigenvalue spread of $\mathbf{R}_{u x}$. We note that increasing the order of the AR model will result in an increase in the complexity of the LMS/Newton Algorithm 1 [7] and hence, the design process will require a trade-off between performance and computational complexity. A theoretical explanation for the impact of nonlinearities on AR modeling is provided in Appendix B.

\section{A. Use of a Highpass Filter to Remove dc Bias}

In this section, we will present a straightforward preprocessing technique to remove the slow modes of convergence that result from using a modified half-wave rectifier. This will enable us to incorporate a nonlinear preprocessor along with the two-channel LMS/Newton algorithm, thereby improving the misalignment, and at the same time, not be concerned about the MSE converging slowly. Furthermore, we will still be able to model the input signals as AR processes having sufficiently low order. In general, this method is applicable to any type of nonlinearity that introduces a dc bias. We have used the example of a modified half-wave rectifier as this transformation is commonly employed in the literature [10], [5].

The discussion in previous section showed that the $\mathrm{dc}$ components introduced through half-wave rectification is the primary reason for the slow convergence of the two-channel LMS/Newton algorithm. The dc bias can be easily suppressed by highpass filtering the nonlinearly transformed input signals $\tilde{x}_{1}(n)$ and $\tilde{x}_{2}(n)$. In practice, the natural loudspeaker response may filter out these dc components introduced via a nonlinearity. But it is important that this bias is removed from the microphone signals before using them as reference inputs to the adaptive filters and we can achieve this by using a simple highpass filter.

In our simulations, we designed a 256-tap highpass filter using a Kaiser window having a cut-off frequency of $100 \mathrm{~Hz}$ and roll-off factor $\beta=0.5$. While these values for the filter parameters represent an ad hoc choice that can be used in practice, the choices of the filter length, the cut-off frequency, the roll-off factor and the windowing function are flexible, as long as the highpass filter serves its primary function of removing the dc components. As before, the length of the modeling adaptive filters $L$ is set equal to 64 and the length of the near-end room echo paths $L_{N}$ is also selected to be the same. The length of the far-end room echo path $L_{F}$ is equal to 128 . The order of the AR model $M$ is chosen to be 8 and $\alpha$ was set to 0.5 . Uncorrelated noise is added to $d(n)$ such that a SNR of $40 \mathrm{~dB}$ is achieved and the simulation results are obtained after averaging over 10 independent runs. Fig. 7(a) compares the MSE and Fig. 7(b) compares the normalized misalignment curves of the LMS/Newton Algorithm 1 with the cases when the modified half-wave rectifier is used and when the same nonlinearity is employed using highpass filtered reference inputs. We can see from the plot of the learning curves that highpass filtering the nonlinearly distorted input signals $\tilde{x}_{1}(n)$ and $\tilde{x}_{2}(n)$ will remove the slow modes of convergence. Furthermore, we still obtain an improvement in the misalignment as compared to the case when no nonlinearity is used.

It is important to note that the highpass filter will only remove the dc bias and the two-channel input signals will still remain sufficiently decorrelated over the remaining frequency spectrum. This can be confirmed by evaluating the coherence values of the reference inputs for the above experiment. The coherence values were estimated as follows [12]:

$$
\begin{aligned}
m_{i} & =\frac{1}{N} \sum_{n=0}^{N-1} \hat{x}_{i}(n), \quad i=1,2 \\
\hat{\gamma} & =\frac{\sum_{n=0}^{N-1}\left[\hat{x}_{1}(n)-m_{1}\right]\left[\hat{x}_{2}(n)-m_{2}\right]}{\sqrt{\sum_{n=0}^{N-1}\left[\hat{x}_{1}(n)-m_{1}\right]^{2} \sum_{n=0}^{N-1}\left[\hat{x}_{2}(n)-m_{2}\right]^{2}}} .
\end{aligned}
$$

$N$ being the length of the data and $\hat{x}_{i}(n)$ denotes the reference inputs to the adaptive filters. Table III lists the condition numbers of the matrix $\mathbf{R}_{u x}$ and the estimated coherence values for the corresponding set of reference inputs. We can see that the highpass filtered reference inputs are just as decorrelated as the original set of nonlinearly distorted input signals. Furthermore, the condition number of $\mathbf{R}_{u x}$ improves significantly upon using a highpass filter. Consequently, the two-channel LMS/ 
TABLE III

CONDITION Numbers of $\mathbf{R}_{u x}$ FOR LMS/NEWTON ALgORITHM 1 AND ESTIMATED COHERENCE OF THE REFERENCE INPUTS

\begin{tabular}{|c|c|c|}
\hline TYPE OF NONLINEARITY & $\chi\left(\mathbf{R}_{u x}\right)$ & ESTIMATED COHERENCE $(\hat{\gamma})$ \\
\hline No nonlinearity & 7.4821 & 0.9999 \\
Modified half-wave rectifier & 50.0659 & 0.9714 \\
Modified half-wave rectifier & 7.0365 & 0.9715 \\
and highpass filtering & & \\
\hline
\end{tabular}

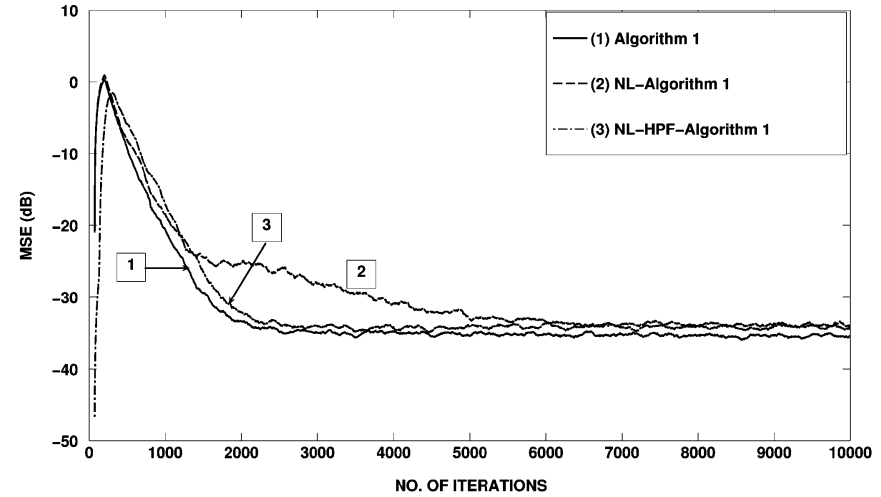

(a)

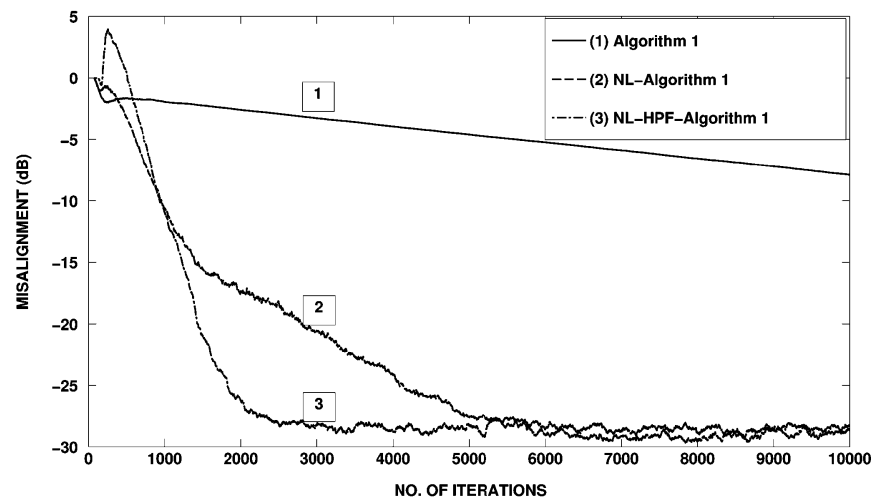

(b)

Fig. 7. (a) Comparison of the MSE. (b) Comparison of the misalignment. (Colored Gaussian noise inputs, $L=64$, Solid-Algorithm 1, Dashed-Algorithm 1 with nonlinearity (26), Dashed-dotted-Algorithm 1 with nonlinearity (26) using highpass filtered reference inputs.)

Newton algorithm implemented using this particular set of reference inputs will not suffer from the slow modes of convergence and will simultaneously benefit from an improvement in the misalignment.

Fig. 8 plots the magnitude of the maximum and minimum eigenvalues of $\mathbf{R}_{u x}$ for various orders of the AR model $M$ and for a fixed length of the modeling adaptive filters $L=$ 64 . Over here, the two-channel stereo input signals were transformed using the modified half-wave rectifier and further preprocessed using a highpass filter. Unlike the earlier result shown in Fig. 6, we can see that the eigenvalue spread is reasonably small for even a low order AR model and it does not reduce drastically upon increasing the order. Thus, using this preprocessing technique will not require us to compromise on the computational complexity by having to increase the order of the AR model for a more accurate computation of $\mathbf{u}(n)$. It would be worthwhile to note that this theory is not applicable to all adaptive algorithms since the main reason for the slow modes of MSE convergence in the LMS/Newton algorithm is the failure

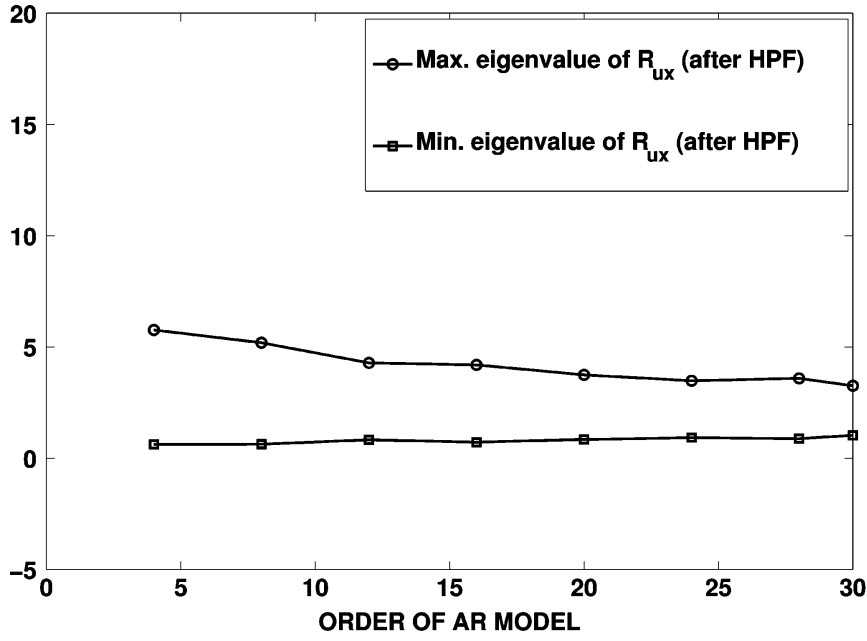

Fig. 8. Variation of the maximum and minimum eigenvalues of $\mathbf{R}_{u x}$ after the use of a highpass filter.

of the AR model based lattice portion of the algorithm in the presence of a dc bias.

Finally, the algorithms are tested to evaluate their effectiveness when speech signals are used as inputs. In this particular set of experiments, $L_{F}$ is set equal to 2048 and $L_{N}$ is selected to be 1024 . We also chose the adaptive filter length $L$ to be 1024 . At a sampling rate of $8 \mathrm{kHz}$, this will enable us to model $128 \mathrm{~ms}$ of echo which is reasonable for a medium-size office room. As before, the order of the AR model $M$ is chosen to be 8 and $\alpha$ was set to 0.5 . Uncorrelated noise is added to $d(n)$ such that a SNR of $40 \mathrm{~dB}$ is achieved and the MSE and normalized misalignment curves obtained after averaging over 10 independent runs are presented in Fig. 9(a) and (b), respectively. From these figures, it is clear that the results are consistent with those for the case when the input is Gaussian and highlight the effectiveness of the proposed solution.

\section{CONCLUSION}

A nonlinear preprocessor can be used in conjunction with the two-channel LMS/Newton algorithm to further reduce the misalignment. However, the addition of certain classes of nonlinearities introduces slow modes in the convergence of the meansquare error. Through analysis of the two-channel LMS/Newton algorithm, a theoretical understanding was provided for the appearance of these slow modes in the presence of nonlinearities. The removal of the dc bias from the nonlinearly distorted reference inputs will enable us to model the input signals as low order AR processes. This will also prevent the two-channel LMS/ Newton algorithm from converging slowly in the mean-square error and the algorithm will simultaneously benefit from a significant improvement in the misalignment. 


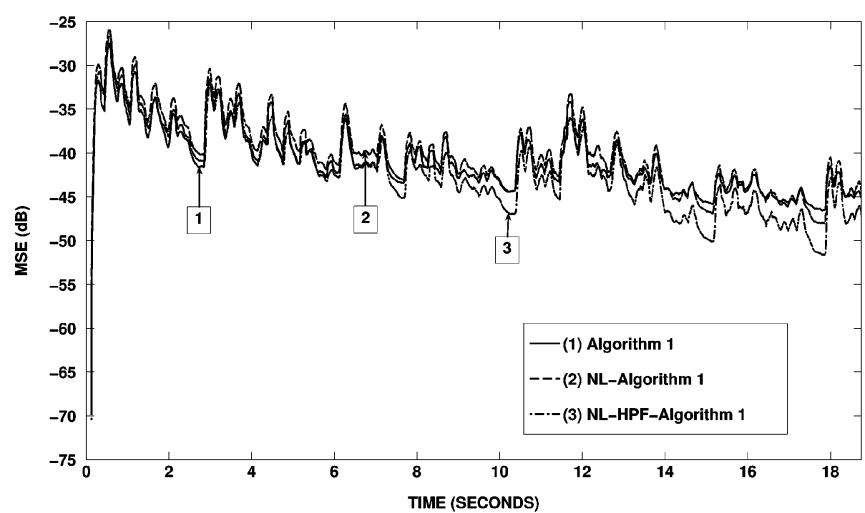

(a)

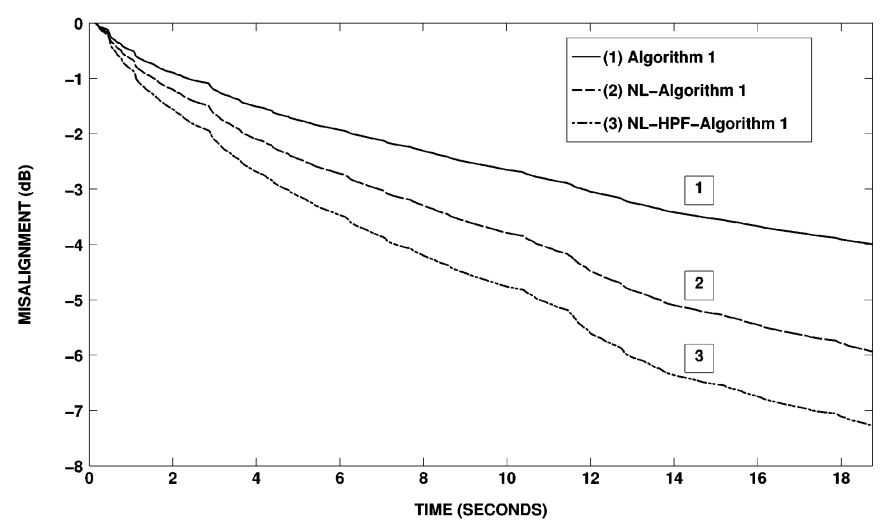

(b)

Fig. 9. (a) Comparison of the MSE. (b) Comparison of the misalignment. (Speech inputs, $L=1024$, Solid-Algorithm 1, Dashed-Algorithm 1 with nonlinearity (26), Dashed-dotted-Algorithm 1 with nonlinearity (26) using highpass filtered reference inputs.)

\section{APPENDIX A}

Evaluation of $E\left[\mathbf{u}(n) \mathbf{x}^{T}(n) \mathbf{K}(n) \mathbf{x}(n) \mathbf{u}^{T}(n)\right]$

First, we note that

$$
\begin{aligned}
& \mathbf{x}^{T}(n) \mathbf{K}(n) \mathbf{x}(n) \\
&= {\left[\mathbf{x}_{1}^{T}(n) \quad \mathbf{x}_{2}^{T}(n)\right]\left[\begin{array}{ll}
\mathbf{K}_{11}(n) & \mathbf{K}_{12}(n) \\
\mathbf{K}_{21}(n) & \mathbf{K}_{22}(n)
\end{array}\right]\left[\begin{array}{c}
\mathbf{x}_{1}(n) \\
\mathbf{x}_{2}(n)
\end{array}\right] } \\
&= \mathbf{x}_{1}^{T}(n) \mathbf{K}_{11}(n) \mathbf{x}_{1}(n) \\
&+\mathbf{x}_{1}^{T}(n) \mathbf{K}_{12}(n) \mathbf{x}_{2}(n)+\mathbf{x}_{2}^{T}(n) \mathbf{K}_{21}(n) \mathbf{x}_{1}(n) \\
&+\mathbf{x}_{2}^{T}(n) \mathbf{K}_{22}(n) \mathbf{x}_{2}(n) .
\end{aligned}
$$

Each of the four terms on the right-hand side of (A1) is a scalar which can be expressed as

$$
\begin{array}{r}
\mathbf{x}_{p}^{T}(n) \mathbf{K}_{p q}(n) \mathbf{x}_{q}(n)=\sum_{i=0}^{L-1} \sum_{j=0}^{L-1} x_{p}(n-i) x_{q}(n-j) k_{p q, i j}(n), \\
\text { for }(p, q)=\{(1,1),(1,2),(2,1),(2,2)\} . \quad \text { (A2) }
\end{array}
$$

In (A2) $k_{p q, i j}(n)$ denotes the $i j$ th element of $\mathbf{K}_{p q}(n)$.

Furthermore, we have

$$
\begin{aligned}
\mathbf{C}(n) & \triangleq \mathbf{u}(n) \mathbf{x}^{T}(n) \mathbf{K}(n) \mathbf{x}(n) \mathbf{u}^{T}(n) \\
& =\left[\begin{array}{l}
\mathbf{u}_{1}(n) \\
\mathbf{u}_{2}(n)
\end{array}\right] \mathbf{x}^{T}(n) \mathbf{K}(n) \mathbf{x}(n)\left[\begin{array}{ll}
\mathbf{u}_{1}^{T}(n) & \mathbf{u}_{2}^{T}(n)
\end{array}\right] \\
& \triangleq\left[\begin{array}{ll}
\mathbf{U}_{11}(n) & \mathbf{U}_{12}(n) \\
\mathbf{U}_{21}(n) & \mathbf{U}_{22}(n)
\end{array}\right] .
\end{aligned}
$$

Thus, $\mathbf{U}_{p q}(n)$, for $(p, q)=\{(1,1),(1,2),(2,1),(2,2)\}$ is a $L \times L$ matrix whose $l m$ th element can be obtained using (A1) and (A2) as

$$
\begin{aligned}
u_{p q, l m}(n)= & u_{p}(n-l) u_{q}(n-m) \\
& \cdot\left\{\sum_{i=0}^{L-1} \sum_{j=0}^{L-1} x_{1}(n-i) x_{1}(n-j) k_{11, i j}(n)\right. \\
& +\sum_{i=0}^{L-1} \sum_{j=0}^{L-1} x_{1}(n-i) x_{2}(n-j) k_{12, i j}(n) \\
& +\sum_{i=0}^{L-1} \sum_{j=0}^{L-1} x_{2}(n-i) x_{1}(n-j) k_{21, i j}(n) \\
& \left.+\sum_{i=0}^{L-1} \sum_{j=0}^{L-1} x_{2}(n-i) x_{2}(n-j) k_{22, i j}(n)\right\} .
\end{aligned}
$$

Taking the expectation of $u_{p q, l m}(n)$, we obtain (A5).

$$
\begin{aligned}
E\left[u_{p q, l m}(n)\right]= & \sum_{i=0}^{L-1} \sum_{j=0}^{L-1} E\left[u_{p}(n-l) u_{q}(n-m)\right. \\
& \left.\cdot x_{1}(n-i) x_{1}(n-j)\right] k_{11, i j}(n) \\
& +\sum_{i=0}^{L-1} \sum_{j=0}^{L-1} E\left[u_{p}(n-l) u_{q}(n-m)\right. \\
& \left.\cdot x_{1}(n-i) x_{2}(n-j)\right] k_{12, i j}(n) \\
& +\sum_{i=0}^{L-1} \sum_{j=0}^{L-1} E\left[u_{p}(n-l) u_{q}(n-m)\right. \\
& \left.\cdot x_{2}(n-i) x_{1}(n-j)\right] k_{21, i j}(n) \\
& +\sum_{i=0}^{L-1} \sum_{j=0}^{L-1} E\left[u_{p}(n-l) u_{q}(n-m)\right. \\
& \left.\cdot x_{2}(n-i) x_{2}(n-j)\right] k_{22, i j}(n) .
\end{aligned}
$$

To simplify our analysis, we assume that the input samples are mutually Gaussian random variables. For a set of real-valued mutually Gaussian random variables $z_{1}, z_{2}, z_{3}$ and $z_{4}$

$$
\begin{aligned}
E\left[z_{1} z_{2} z_{3} z_{4}\right]= & E\left[z_{1} z_{2}\right] E\left[z_{3} z_{4}\right] \\
& +E\left[z_{1} z_{3}\right] E\left[z_{2} z_{4}\right]+E\left[z_{1} z_{4}\right] E\left[z_{2} z_{3}\right] .
\end{aligned}
$$

We also make note of the fact that

$E\left[u_{p}(n-l) x_{q}(n-i)\right]= \begin{cases}1 ; & \text { if } l=i \text { and } p=q \\ 0 ; & \text { otherwise }\end{cases}$

and

$$
\begin{array}{ll}
\mathbf{K}_{p q}=\mathbf{K}_{p q}^{T} ; & \text { for }(p, q)=\{(1,1),(2,2)\} \\
\mathbf{K}_{p q}=\mathbf{K}_{q p}^{T} ; & \text { for }(p, q)=\{(1,2),(2,1)\} .
\end{array}
$$

Then, using (A6), (A7), and (A8), we can simplify (A5) as

$$
\begin{aligned}
E\left[u_{p q, l m}(n)\right]= & r_{u_{p} u_{q}}^{l m} \sum_{i=0}^{L-1} \sum_{j=0}^{L-1}\left\{r_{x_{1} x_{1}}^{i j} k_{11, i j}(n)\right. \\
& +r_{x_{1} x_{2}}^{i j} k_{12, i j}(n)+r_{x_{2} x_{1}}^{i j} k_{21, i j}(n) \\
& \left.+r_{x_{2} x_{2}}^{i j} k_{22, i j}(n)\right\}+2 k_{p q, l m}(n) .
\end{aligned}
$$


where $r_{u_{p} u_{q}}^{l m}$ and $r_{x_{p} x_{q}}^{i j}$ are the $l m$ th and $i j$ th elements of the correlation matrices $\mathbf{R}_{u_{p} u_{q}}$ and $\mathbf{R}_{x_{p} x_{q}}$, respectively for $(p, q)=$ $\{(1,1),(1,2),(2,1),(2,2)\}$. (A9) can be further simplified to

$$
\begin{aligned}
E\left[u_{p q, l m}(n)\right]= & r_{u_{p} u_{q}}^{l m}\left\{\operatorname{tr}\left[\mathbf{K}_{11} \mathbf{R}_{x_{1} x_{1}}\right]+\operatorname{tr}\left[\mathbf{K}_{12} \mathbf{R}_{x_{1} x_{2}}\right]\right. \\
& \left.+\operatorname{tr}\left[\mathbf{K}_{21} \mathbf{R}_{x_{2} x_{1}}\right]+\operatorname{tr}\left[\mathbf{K}_{22} \mathbf{R}_{x_{2} x_{2}}\right]\right\} \\
& +2 k_{p q, l m}(n)
\end{aligned}
$$

where $\operatorname{tr}[\cdot]$ denotes the sum of the diagonal elements of the corresponding matrix.

We further take note that

$$
\begin{aligned}
\operatorname{tr}\left[\mathbf{K}(n) \mathbf{R}_{x x}(n)\right] & =\operatorname{tr}\left[\mathbf{K}_{11} \mathbf{R}_{x_{1} x_{1}}\right]+\operatorname{tr}\left[\mathbf{K}_{12} \mathbf{R}_{x_{2} x_{1}}\right] \\
& +\operatorname{tr}\left[\mathbf{K}_{21} \mathbf{R}_{x_{1} x_{2}}\right]+\operatorname{tr}\left[\mathbf{K}_{22} \mathbf{R}_{x_{2} x_{2}}\right] .
\end{aligned}
$$

Hence, (A10) can be rewritten as

$$
\begin{aligned}
E\left[u_{p q, l m}(n)\right]= & r_{u_{p} u_{q}}^{l m}\left\{\operatorname{tr}\left[\mathbf{K}(n) \mathbf{R}_{x x}(n)\right]\right. \\
& \left.+\operatorname{tr}\left[\Delta \mathbf{K}(n) \Delta \mathbf{R}_{x x}\right]\right\}+2 k_{p q, l m}(n)
\end{aligned}
$$

where $\Delta \mathbf{K}(n)=\mathbf{K}_{12}(n)-\mathbf{K}_{21}(n)$ and $\Delta \mathbf{R}_{x x}=\mathbf{R}_{x_{1} x_{2}}-$ $\mathbf{R}_{x_{2} x_{1}}$.

Finally, combining these elements to construct the matrix $E[\mathbf{C}(n)]=E\left[\mathbf{u}(n) \mathbf{x}^{T}(n) \mathbf{K}(n) \mathbf{x}(n) \mathbf{u}^{T}(n)\right]$ as given by (A3), we get (19).

\section{APPENDIX B}

IMPACT OF NONLINEARITIES ON AUTOREGRESSIVE MODELING OF RANDOM PROCESSES

A random process $x(n)$ is said to be autoregressive (AR) of order $M$ if it satisfies the difference equation

$$
x(n)-\sum_{i=1}^{M} h_{i} x(n-i)=\nu(n)
$$

where $h_{i}$ represents the AR coefficients and $\nu(n)$ is a zero-mean white noise process called the innovation of $x(n)$. It can be shown that the set of forward prediction coefficients of $x(n)$, denoted by $a_{M, i}, i=1,2, \ldots, M$ match the AR coefficients $h_{i}$. In particular, if a predictor of order $M^{\prime} \geq M$ is used, then we have [9]

$$
a_{M^{\prime}, i}= \begin{cases}h_{i} ; & 1 \leq i \leq M \\ 0 ; & M+1 \leq i \leq M^{\prime}\end{cases}
$$

Before we proceed further, we define the autocorrelation function of the input process for lag $k$ as

$$
r_{x x}(k)=E[x(n) x(n-k)] .
$$

It will also be useful to note that, for an AR process, as $k \rightarrow$ $\infty, r_{x x}(k) \rightarrow 0$ [9].

We recognize that modeling of an AR process $x(n)$ is a conventional filtering problem. The optimal forward prediction coefficients, $\mathbf{a}_{M}=\left[a_{M, 1}, a_{M, 2}, \ldots, a_{M, M}\right]^{T}$ can be obtained as

$$
\mathbf{a}_{M}=\mathbf{R}^{-1} \mathbf{r}
$$

where

$$
\mathbf{R}=\left[\begin{array}{cccc}
r_{x x}(0) & r_{x x}(1) & \cdots & r_{x x}(M-1) \\
r_{x x}(1) & r_{x x}(2) & \cdots & r_{x x}(M-2) \\
\vdots & \vdots & \ddots & \vdots \\
r_{x x}(M-1) & r_{x x}(M-2) & \cdots & r_{x x}(0)
\end{array}\right]
$$

and

$$
\mathbf{r}=\left[\begin{array}{c}
r_{x x}(1) \\
r_{x x}(2) \\
\vdots \\
r_{x x}(M)
\end{array}\right]
$$

Now, if we modify $x(n)$ with a nonlinear transformation as

$$
x^{\prime}(n)=x(n)+\alpha \tilde{x}(n)
$$

where $\alpha$ controls the amount of nonlinearity added and $\tilde{x}(n)$ is the nonlinearity, then it follows from (B3) and (B7) that the autocorrelation function of the modified input process is

$$
r_{x^{\prime} x^{\prime}}(k)=r_{x x}(k)+\alpha r_{\tilde{x} x}(k)+\alpha r_{x \tilde{x}}(k)+\alpha^{2} r_{\tilde{x} \tilde{x}}(k) .
$$

If we assume $x(n)$ to be a stationary Gaussian process with variance $\sigma^{2}$, then we can use Price's theorem [14] to obtain the cross-correlation functions

$$
r_{\tilde{x} x}(k)=r_{x \tilde{x}}(k)=K_{p} r_{x x}(k)
$$

where the constant

$$
K_{p}=\frac{1}{\sqrt{2 \pi} \sigma^{3}} \int_{-\infty}^{\infty} x \tilde{x} e^{-\left(\frac{x^{2}}{2 \sigma^{2}}\right)} d x .
$$

Using (B9), we rewrite (B8) as

$$
r_{x^{\prime} x^{\prime}}(k)=r_{x x}(k)\left[1+2 \alpha K_{p}\right]+\alpha^{2} r_{\tilde{x} \tilde{x}}(k) .
$$

$K_{p}$ and $r_{\tilde{x} \tilde{x}}(k)$ have been evaluated for several nonlinearities in [13], [15].

We will now examine the autocorrelation function $r_{x^{\prime} x^{\prime}}(k)$ when the input process $x(n)$ is modified using two types of nonlinearities.

Case 1: For $\tilde{x}(n)=|x(n)|$,

$$
K_{p}=0,
$$

$r_{\tilde{x} \tilde{x}}(k)=\frac{2 \sigma^{2}}{\pi}\left\{\frac{r_{x x}(k)}{\sigma^{2}} \sin ^{-1}\left(\frac{r_{x x}(k)}{\sigma^{2}}\right)+\sqrt{1-\frac{r_{x x}^{2}(k)}{\sigma^{4}}}\right\}$.

Then, using (B11), we have

$$
\begin{aligned}
& r_{x^{\prime} x^{\prime}}(k) \\
& =r_{x x}(k)\left\{1+\frac{2 \alpha^{2}}{\pi} \sin ^{-1}\left(\frac{r_{x x}(k)}{\sigma^{2}}\right)\right\}+\underbrace{\frac{2 \alpha^{2} \sigma^{2}}{\pi} \sqrt{1-\frac{r_{x x}^{2}(k)}{\sigma^{4}}}}_{\text {bias }} .
\end{aligned}
$$




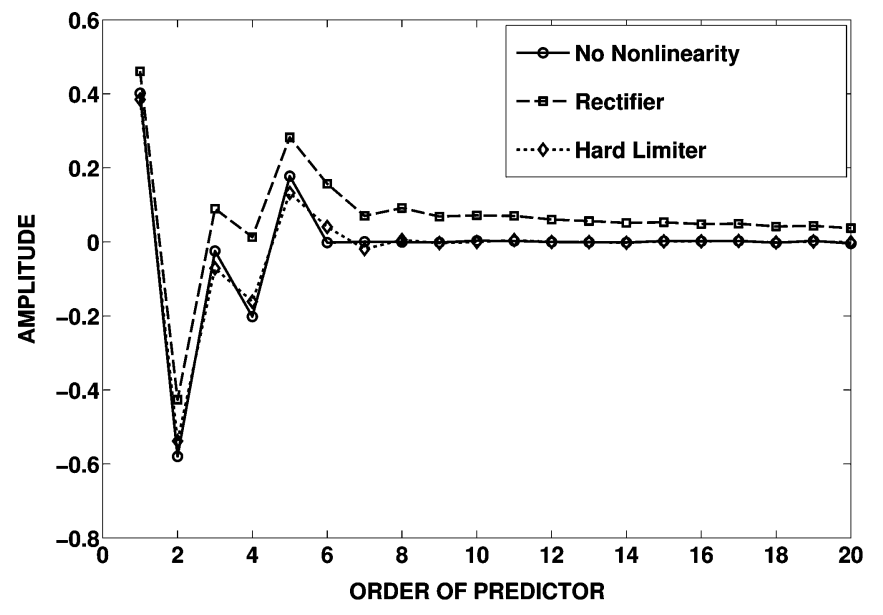

Fig. 10. Partial correlation coefficients.

Case 2: For $\tilde{x}(n)=\operatorname{sgn}(x(n))$,

$$
\begin{aligned}
K_{p} & =\frac{1}{\sigma} \sqrt{\frac{2}{\pi}}, \\
r_{\tilde{x} \tilde{x}}(k) & =\frac{2}{\pi} \sin ^{-1}\left(\frac{r_{x x}(k)}{\sigma^{2}}\right) .
\end{aligned}
$$

Once again, using (B11), we have

$$
\begin{aligned}
r_{x^{\prime} x^{\prime}}(k) & r_{x x}(k)\left\{1+\frac{2 \alpha}{\sigma} \sqrt{\frac{2}{\pi}}\right\}+\underbrace{\frac{2 \alpha^{2}}{\pi} \sin ^{-1}\left(\frac{r_{x x}(k)}{\sigma^{2}}\right)}_{\text {bias }} .
\end{aligned}
$$

Depending upon the choice of nonlinearity, the matrix $\mathbf{R}$ and the vector $\mathbf{r}$ can be constructed using either (B13) or (B15), and the optimal predictor coefficients can be obtained using (B4).

A careful examination of (B13) will reveal that $r_{x^{\prime} x^{\prime}}(k)$ will remain biased even as $k \rightarrow \infty$. Consequently, the forward prediction coefficients obtained using (B4) will be biased as well. Furthermore, we may never be able obtain a set of predictor coefficients that will satisfy (B2). In other words, we may require infinite coefficients to model this particular input process as AR. However, for the autocorrelation function presented in (B15), the bias term will vanish as $k \rightarrow \infty$. This will allow us to obtain a set of finite prediction coefficients that will satisfy (B2) closely.

We will use a simple simulation example to further validate our observations. The input signal $x(n)$ was modeled as a fifthorder AR process with the poles of the transfer function located at $0.5,0.85 e^{ \pm j \pi / 3}$ and $0.7 e^{ \pm j 2 \pi / 3}$ [9]. The same input signal was also modified using the above nonlinear transformations, wherein, $\alpha$ was set to 0.5 . The predictor coefficients were computed using (B4) for each set of inputs and the Levinson-Durbin algorithm was used to convert these coefficients into a set of partial correlation (PARCOR) coefficients [9]. The maximum order of the predictor was chosen to be 20 and the computed PARCOR coefficients are shown in Fig. 10. The property of the PARCOR coefficients ensures that once they converge to zero, the prediction-error cannot be reduced further upon increasing the predictor order [9]. When nonlinearity is not used or upon using a hard limiter, the PARCOR coefficients converges to zero for low predictor orders. However, the rectifier causes the PARCOR coefficients to converge very slowly and we may have to choose a very high predictor order in order to minimize the mean-square of the prediction-error. This behavior of the PARCOR coefficients is reflected in the LMS/Newton algorithm and thereby, justifies the need for increasing the order of the AR model.

\section{ACKNOWLEDGMENT}

The authors would like to thank the anonymous reviewers whose constructive comments and suggestions greatly helped to improve the quality of the paper.

\section{REFERENCES}

[1] M. M. Sondhi, D. R. Morgan, and J. L. Hall, "Stereophonic acoustic echo cancellation-An overview of the fundamental problem," IEEE Signal Process. Lett., vol. 2, no. 8, pp. 148-151, Aug. 1995.

[2] J. Benesty, T. Gänsler, D. M. Morgan, M. M. Sondhi, and S. L. Gay, Advances in Network and Acoustic Echo Cancellation. Berlin, Germany: Springer-Verlag, 2001.

[3] E. Hänsler and G. Schmidt, Topics in Acoustic Echo and Noise Control. Berlin, Germany: Springer-Verlag, 2006.

[4] J. Benesty, D. R. Morgan, and M. M. Sondhi, "A better understanding and an improved solution to the specific problems of stereophonic acoustic echo cancellation," IEEE Trans. Speech Audio Process., vol. 6, no. 2, pp. 156-165, Mar. 1998.

[5] A. W. H. Khong and P. A. Naylor, "Stereophonic acoustic echo cancellation employing selective-tap adaptive algorithms," IEEE Trans. Audio, Speech, Lang. Process., vol. 14, no. 3, pp. 785-796, May 2006.

[6] T. Hoya, Y. Loke, J. A. Chambers, and P. A. Naylor, "Application of the leaky extended LMS (XLMS) algorithm in stereophonic acoustic echo cancellation," Signal Process., vol. 64, no. 1, pp. 87-91, Jan. 1998.

[7] H. I. K. Rao and B. Farhang-Boroujeny, "Fast LMS/Newton algorithms for stereophonic acoustic echo cancellation," IEEE Trans. Signal Process., vol. 57, no. 8, pp. 2919-2930, Aug. 2009.

[8] B. Farhang-Boroujeny, "Fast LMS/Newton algorithms based on autoregressive modeling and their application to acoustic echo cancellation," IEEE Trans. Signal Process., vol. 45, no. 8, pp. 1987-2000, Aug. 1997.

[9] B. Farhang-Boroujeny, Adaptive Filters: Theory and Applications. Chichester, U.K.: Wiley, 1998.

[10] K. Mayyas, "Stereophonic acoustic echo cancellation using lattice orthogonalization," IEEE Trans. Speech Audio Process., vol. 10, no. 7, pp. 517-525, Oct. 2002.

[11] S. Haykin, Adaptive Filter Theory. New Delhi, India: Pearson Education, 2003.

[12] T. Gänsler and J. Benesty, "New insights into the stereophonic acoustic echo cancellation problem and an adaptive nonlinearity solution," IEEE Trans. Speech Audio Process., vol. 10, no. 5, pp. 257-267, Jul. 2002.

[13] D. R. Morgan, J. L. Hall, and J. Benesty, "Investigation of several types of nonlinearities for use in stereo acoustic echo cancellation," IEEE Trans. Speech Audio Process., vol. 9, no. 6, pp. 686-696, Sep. 2001.

[14] A. Papoulis, Probability, Random Variables and Stochastic Processes. New York: McGraw-Hill, 1984.

[15] R. F. Baum, "The correlation function of Gaussian noises passed through nonlinear devices," IEEE Trans. Inf. Theory, vol. IT-15, pp. 448-456, Jul. 1969.

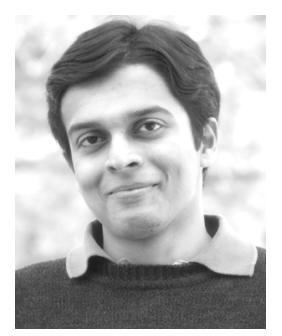

Harsha I. K. Rao (S'06) received the B.E. degree in electronics and communication engineering with highest honors from the National Institute of Technology, Tiruchirappalli, India, in 2003 and the M.E. and Ph.D. degrees in electrical engineering from the University of Utah, Salt Lake City, in 2008, and 2010, respectively. His dissertation focused on the problems of acoustic crosstalk cancellation and stereophonic acoustic echo cancellation to achieve sound spatialization using a pair-wise loudspeaker paradigm.

From 2003 to 2004, he was a Design Engineer at ABB, Bangalore, India. Since 2010, he has been with HARMAN, Salt Lake City, UT, where he has worked on the analysis and development of DSP algorithms and products. His research interests include adaptive filtering and its application in audio signal processing. 


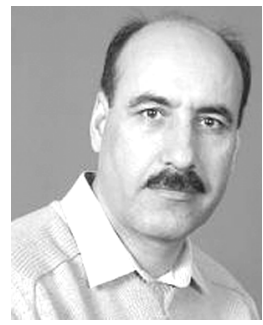

Behrouz Farhang-Boroujeny (M'84-SM'90) received the B.Sc. degree in electrical engineering from Teheran University, Iran, in 1976, the M.Eng. degree from the University of Wales Institute of Science and Technology, U.K., in 1977, and the Ph.D. degree from Imperial College, University of London, U.K., in 1981.

From 1981 to 1989 , he was with the Isfahan University of Technology, Isfahan, Iran. From 1989 to 2000, he was with the National University of Singapore. Since August 2000, he has been with the University of Utah, Salt Lake City, where he is currently a Professor and Associate Chair of the department. He is an expert in the general area of signal processing. His current scientific interests are adaptive filters, multicarrier communications, detection techniques for space-time coded systems, cognitive radio, and signal processing applications to optical devices. In the past, he has worked and has made significant contribution to areas of adaptive filters theory, acoustic echo cancellation, magnetic/optical recoding, and digital subscriber line technologies. He is the author of the books Adaptive Filters: Theory and Applications (Wiley, 1998) and Signal Processing Techniques for Software Radios (self-published, Lulu Publishing, 2009).

Dr. Farhang-Boroujeny received the UNESCO Regional Office of Science and Technology for South and Central Asia Young Scientists Award in 1987. He served as Associate Editor of the IEEE TRANSACTIONS ON SIGNAL PROCESSING from July 2002 to July 2005. He is now serving as Associate Editor of the IEEE SignAl PROCESSING LETTERS. He has also been involved in various IEEE activities, including the chairmanship of the Signal Processing/Communications Chapter of IEEE of Utah in 2004 and 2005. 